This item was submitted to Loughborough's Research Repository by the author.

Items in Figshare are protected by copyright, with all rights reserved, unless otherwise indicated.

\title{
Tactile sensors for robot handling
}

PLEASE CITE THE PUBLISHED VERSION

PUBLISHER

Professional Engineering Publishing / @ IMECHE

VERSION

VoR (Version of Record)

LICENCE

CC BY-NC-ND 4.0

REPOSITORY RECORD

Foroughi, F., Homer Rahnejat, and H. Bera. 2019. "Tactile Sensors for Robot Handling”. figshare. https://hdl.handle.net/2134/4811. 
This item was submitted to Loughborough's Institutional Repository (https://dspace.lboro.ac.uk/) by the author and is made available under the following Creative Commons Licence conditions.

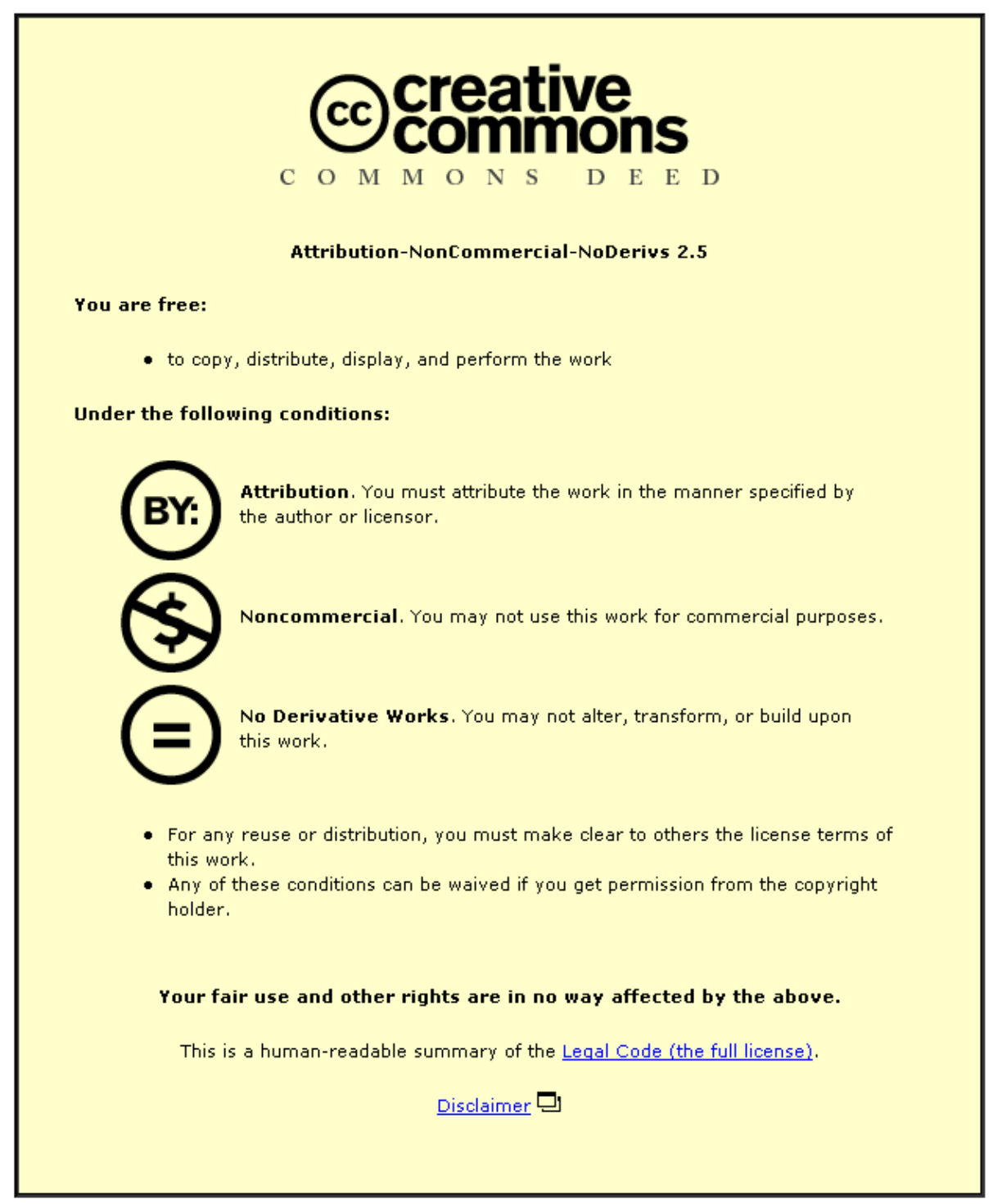

For the full text of this licence, please go to: http://creativecommons.org/licenses/by-nc-nd/2.5/ 


\section{Tactile sensors for robot handling}

F Foroughi, BSc, H Rahnejat,* BSc, MSc, PhD, DIC, AMIMechE and H Bera, MSc, PhD, CEng, MIProdE, MIQA

Manufacturing and Robotics Centre, Department of Mechanical and Production Engineering,

South Bank Polytechnic, Borough Road, London SE1

First and second generation robots have been used cost effectively in high-volume 'fixed' or 'hard' automated manufacturing/assembly systems. They are 'limited-ability' devices using simple logic elements or primitive sensory feedback. However, in the unstructured environment of most manufacturing plants it is often necessary to locate, identify, orientate and position randomly presented components.

Visual systems have been researched and developed to provide a coarse resolution outline of objects. More detailed and precise definition of parts is usually obtained by high resolution tactile sensing arrays. This paper reviews and discusses the current state of the art in tactile sensing.

\section{INTRODUCTION}

Industrial robots have been in use since the early 1960 s. The 'first generation' robots were employed in machine loading and material transfer operations. Gradually, more complex tasks were included such as palletizing, welding, painting, application of adhesives etc. For these applications 'second generation' robots with limited sensory preception are required to interact with the environment.

In fact first and second generation robots are used for tasks that fall into two broad categories. Firstly, work that is hazardous in nature or is to be performed in hostile surroundings (1). Secondly, operations that are relatively simple and highly repetitive (2). These tasks are conducive to operator fatigue and result in imprecise actions. Many of these tasks can be accomplished by 'pick and place' robots with no sensory feedback. For some other applications such as arc welding or simple assembly work primitive sensing configurations suffice. In all cases robots are primarily used to replace the human operators in order to enhance operational integrity in highly repetitive manufacture and process a large volume output (for example round the clock production).

A major prerequisite in the design of these systems is the relative simplicity of the tasks to be accomplished. Therefore, ease of handling for robots must be ensured through proper provision for part presentation, tool deployment and dedicated programming (3).

However, if industrial robots are to make an impact in manufacturing, it is generally accepted that they should possess a capacity to perceive and interact with the surrounding unstructured environments $(4,5)$. They should be able to search, detect, recognize, orientate and grasp randomly presented parts (for example from a bin). Furthermore, move, place, join, insert, fit, align, screw and turn parts safely in collision-free movements. 'Third generation' robots have been developed to perform automatically these tasks in an unstructured environment. These are reprogrammable sensor-

\footnotetext{
The MS was received on 21 July 1986 and was accepted for publication on 18 November 1986.

* Present address: Advanced Automation Laboratory, School of Mechanical, Aeronautical and Production Engineering, Kingston Polytechnic, Kingston,
} Surrey. mediated robots which are computer-controlled and emulate the actions of a trained human operator (4).

Trained human operators possess a comprehensive sensory feedback system which monitors a large amount of information in various forms. These include visual, sound, smell, tactile, taste and range data. Therefore, a human operator is far better equipped and infnitely more versatile than substitute robots. To be economically viable, reprogrammable robotic systems must perform specific tasks as well or better than human workers. Therefore, for particular applications they must be reliable and fast in comparison with human operators.

\section{SENSORS FOR ROBOTIC APPLICATIONS}

Present day industrial robots (being of first and second generation) are unable to adopt to random positions and orientation of parts. This limits their use in material handling and assembly related operations as well as their ability to emulate jobs that are presently accomplished by skilled operators $(5,6)$. Therefore, it is necessary to introduce sensory perception for industrial robots in order to enhance their manipulative capabilities.

There are various sensing configurations that can be employed to perform qualitative and quantitative inspection of environmental tasks. Sensors can be broadly divided into two groups:

(a) contact sensors;

(b) non-contact sensors.

Each group can be further subdivided into classifications according to the physical properties that are sensed.

The contact sensors consist of devices that monitor changes perceived by touch, pressure, force and temperature. Complex configurations of some of these sensors can replace vision rather like the enhanced sensing ability of blind people. Notably high-resolution tactile arrays can be developed to replace vision where the latter is found to be unsuitable for a particular application. In many cases tactile array sensors are used to complement vision (7). 
The non-contact sensors measure the response of a target to some form of radiation such as light, X-rays, infra-red, radar, acoustics etc.

\section{PROXIMITY SENSORS AND VISION SYSTEMS}

The most widely used non-contact sensors are proximity devices which indicate the presence of an object within a fixed space from the manipulator. There are many types of proximity sensors that have been developed. These include Hall-effect and eddy current sensors which are used to maintain a constant gap between the manipulator and the object being sensed (8). Other types of proximity sensors are ultrasonic, solid-state photoelectric configurations, magnetic bridges and radio-frequency devices. Photoelectric configurations are most widely utilized proximity devices and consist of solid-state light emitting diodes (LED), transmitting infra-red light which is received by solidstate photodiodes (9) (Fig. 1). The main drawbacks of this sensor are its low sensitivity, problems associated with reflectance, detection of curved profiles and surface irregularities of intruding objects, general requirements for high levels of illumination and expensive arrays. Nevertheless, modern solid-state sensors have been deployed increasingly in part recognition and presentation applications. In most of these applications photoelectric sensors are used as an integral part of a hand-eye co-ordinating system for simple object manipulation (9-12).

The work reported in reference (9) involves the recognition of flat workpieces from a top-view image processed by a TV camera. The camera and a manipulator are both mounted on a turret. After the job of workpiece identification and orientation is accomplished, the manipulator is rotated into a position

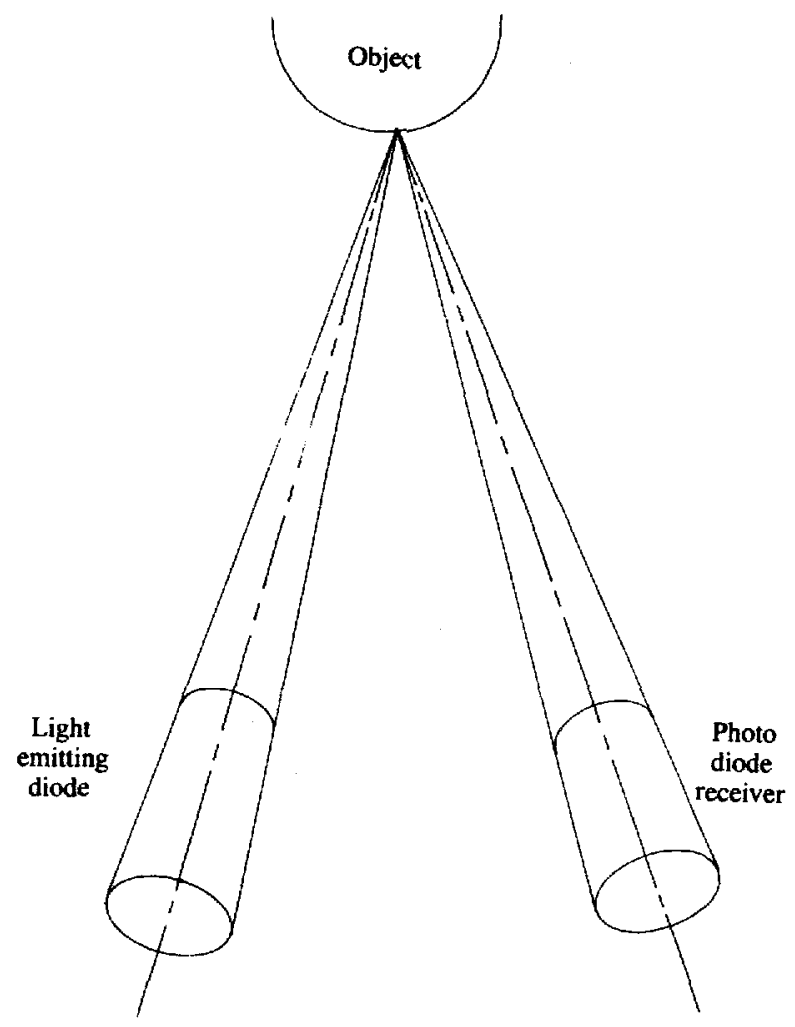

Fig. 1 A photoelectric proximity sensor coaxial with the original optical axis of the camera and grasps the workpiece. Reference (11) describes a recognition system controlled by a minicomputer to identify and determine the position and orientation of randomly placed objects on a table. A workpiece is recognized by using either of two methods. One method is based on sequential recording of the minimum number of features that can distinguish one object from another. The other method involved the measurement of all features corresponding to a particular workpiece.

Similar recognition procedures are adopted by other workers (13-16). For example, reference (12) employs multiple infra-red LEDs attached to a rectangular plate on a robotic manipulator and are turned sequentially with the intensity controlled by a microprocessor. A power spectral density camera is used to receive the emitting light from these LEDs and detect the brightest spot in the image. The corresponding analogue output is converted to digital data which are transferred to the microprocessor. A novel use of multiple photoelectric proximity sensors is presented in reference (17) where two sensors are used to centre the position of a manipulator over the highest point of an object.

In general the goal of vision systems is to recognize randomly presented parts in unstructured environments. The human visual system creates a detailed surface description of its surroundings including depth and orientation information at all points. High resolution information of this type is beyond the capability of present machine vision systems. Therefore, visual data must be supplemented by high-resolution surface description of objects gathered by tactile sensing arrays. The intention in this paper is to provide a detailed description of parameters investigated for tactility and configurations that are employed for this purpose. In doing so, special attention is paid to their suitable applications and their limitations.

\section{TACTILE AND TOUCH SENSING CONFIGURATIONS}

Vision sensing systems are used to provide part position and pose data to the robot end-effector. Any obstructions or occlusions in the field of vision system hinder the gathering of information and can lead to recording of incorrect data. In addition, high-resolution visual data collection requires a good degree of illumination that is not always possible or is expensive. Also, information about inaccessible areas may be required, for example, to carry out some repair work. Such applications impose severe limitations in the use of vision systems. Tactile sensing arrays avoid these problems because all of the data acquisition takes place at the interface between the end-effector and the workpiece.

The term tactile sensing is defined as continuous variable sensing of force, pressure, torque, compliance or slip. This type of system operates in a similar manner to the human sense of tactility. The human sense of feel works as a series of electrical nerve impulses which are transmitted by a network of nerves between the fingers and the brain. To emulate this, tactile sensing arrays must possess skin-like properties capable of reporting graded signals and parallel patterns of touching (18).

In contrast simple touch sensors are primitive devices which are used to stop the motion of a robot when its 
end-effector makes contact with an intruding object in its path. These devices are employed for applications like detection of objects in the robot path, collision avoidance and coarse resolution object recognition (19). Simple touch sensors involve monitoring of binary or continuously variable parameters from a single point contact or just a few points. Simple microswitches or on-off switches detect a contact or poke-probe travel can be used to provide force or pressure feedback. Operating threshold binary signals are utilized in many cases using pressure sensors of conductive material (20), pneumatic threshold sensors (21) or metal contact detectors. Detection of displacement and force by strain gauges $(\mathbf{2 2}, \mathbf{2 3})$, potentiometers $(\mathbf{2 4})$ or gyroscopes are also utilized.

\section{REQUIREMENTS FOR VIABLE TACTILE SENSING}

There are two basic desirable properties of tactile sensing that are frequently cited by many researchers. These are:

(a) skin-like properties;

(b) hand-like manipulation.

The former refers to emulation of the human sense of feel. It envisages the development of variable skin compliant capability, perhaps in the form of distributed arrays of sensing transducers on thin, flexible substrates. Alternatively rigid sensing surfaces may be employed and compliance is built into the controlling software (18). Obviously there are a number of design specifications which should be adhered to in the development of touch transducing configurations. These relate to the spatial density of sensing arrays or matrices as well as suitable materials that can withstand the intended working environment. Additionally, a fair amount of preprocessing or programming is required to provide an element of intrinsic intelligence. The characteristics of the sensing elements should include high sensitivity, fast response and continuous variable output. Other considerations in design are the cost of materials, input power, programming, maintenance and a high level of operational and technical reliability. A good example of a high-resolution tactile sensor incorporating the above mentioned design considerations is the work reported in reference (25) where a compliant soft membrane is used in conjunction with a light source (Fig. 2). When a contact is made between the membrane and an object the air gap is bridged and refraction of light rays are sensed by a solid-state camera, giving a high-resolution map of the contact domain. The shape of the contact determines the outline of the object and its pose. Another simpler (thus more reliable) configuration is being researched by the authors. In this case transduction is based on the variation of an array of pneumatic back-pressures sensed by a corresponding array of integrated circuit miniature pressure sensors, maintained at a constant low-voltage supply (Fig. 3). The air supply pressure is $5 \mathrm{lbf} / \mathrm{in}^{2}$ through each hole of $2 \mathrm{~mm}$ diameter with inter-hole spacing of $2 \mathrm{~mm}$. (No compliant substrate is necessary as active sensor-mediation is being developed by Software Controls.)

The sensing devices should be placed in a desired position so that the robot gripper can manipulate objects in a hand-like manner. Therefore, flexible jointed fingers of great dexterity are required. Human operators, by experience, acquire the skill needed to effect precision assembly operations. This experience is supplemented by the sensitivity and flexibility of the human hand and wrist. Multi-finger grippers having multitudes of joints, incorporating appropriate sensing devices, can discriminate between randomly presented objects and manipulate them in a dexterous manner. Parameters such as force, torque, slip and pressure are exploited to determine safe (optimal) handling of parts.

\section{SLIP DETECTION DEVICES}

Hand-like manipulation calls for a flexible handling capacity. This means that a stable grasping force must be achieved for a variety of objects of various shapes, sizes and textures. Therefore, the gripping force has to be variable depending on the application, and in particular adapted to changes in feedback provided by the employed sensors. Most industrial robots, however, utilize a constant gripping force and as such lack the flexibility and control that can be exercised by a 'feedback-assisted' gripper actuating mechanism.

One way of performing stable and flexible material handling is through detection of slip. In this way the optimum grasping force application can be determined

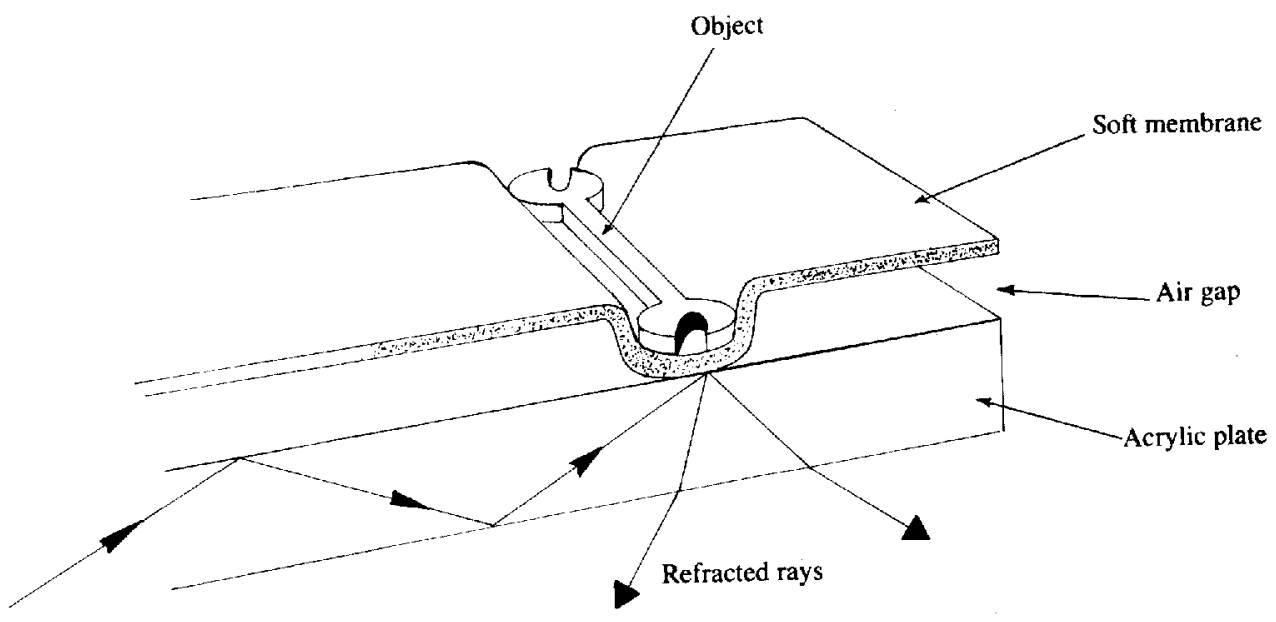

Fig. 2 A variable pressure to light converting sensor 


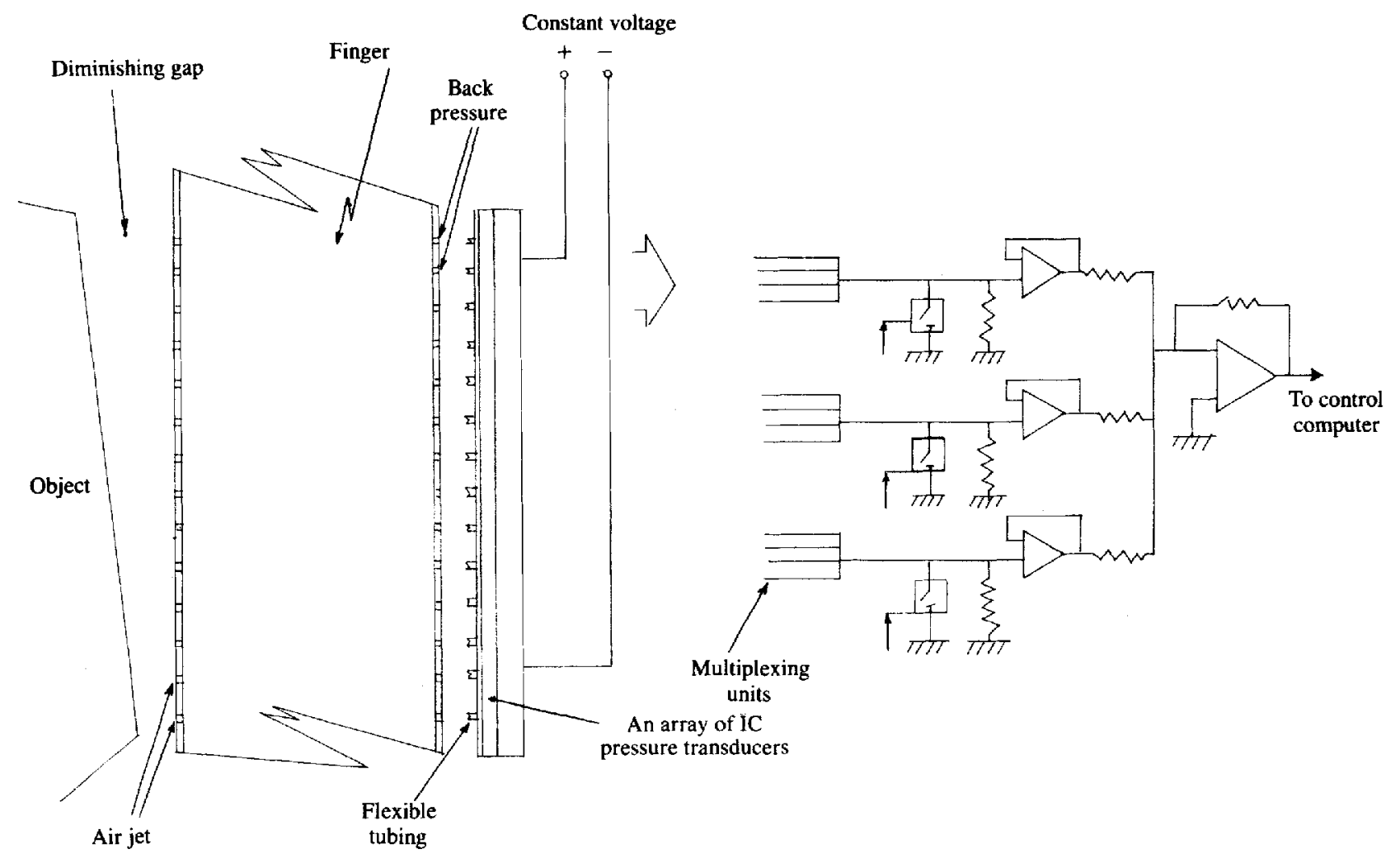

Fig. 3 A high-resolution pneumatic tactile sensing system

by an adaptive preprocessed slip measuring sensing system. There are three main methods of slip detection:

(a) small displacement sensing on a grasping plate or a jaw (26);

(b) transduction of vibrations produced by the slip of a finger surface;

(c) estimation of slip by the exerted pressure levels on a grasping jaw or a finger tip.

Masuda et al. (26) describe a slip sensor which detects the slip displacement through the rotating motion of rollers that contact an object. A roller is employed which is attached to a finger through a leaf spring. The attachment ensures that the roller protrudes out of the end-effector finger surface by $1 \mathrm{~mm}$ when the finger is in the open position. When the finger is closed in grasping an object the roller retreats to the surface of the finger and aids in the support of the object. The rotating angle that the roller goes through in doing so is detected and provides a quantitative measure of slip. Figure 4 illustrates a simple sketch of this type of device.

The slip detecting system in reference (26) uses a slitted disc which is attached to the inner wall of the roller shown in Fig. 4. The rotating angle of the slitted disc is measured by an LED and a phototransistor attached to the roller shaft and corresponds directly to the amount of slip between the object and the robot end-effector. The grasping force compensation system consists of two main parts:

(a) the slip sensor;

(b) the grasping force controller with pressure sensor.

Two other slip sensing configurations are presented in references (27) and (28). The first type is a simple slip sensor consisting of a digital magnetic sensing element and a non-magnetic brass sphere with embedded steel pins. A narrow gap is incorporated between the sphere and a magnetic head. The sphere is supported by a ring and sticks out 1-2 mm from the support by a structure opposite the magnetic head. When an object in contact with the sphere slips, the rolling motion of the latter generates electrical pulses corresponding to the alternate traverse of non-magnetic and magnetic points of sphere past the magnetic head. This device enables the detection of slip but no information is collected with regard to the direction or amount of slip involved.

The second device reported in reference (28) consists of 16 electrical contact points distributed evenly around a circular plate attached to a needle. Therefore, a directional slip detecting sensor is developed which allows

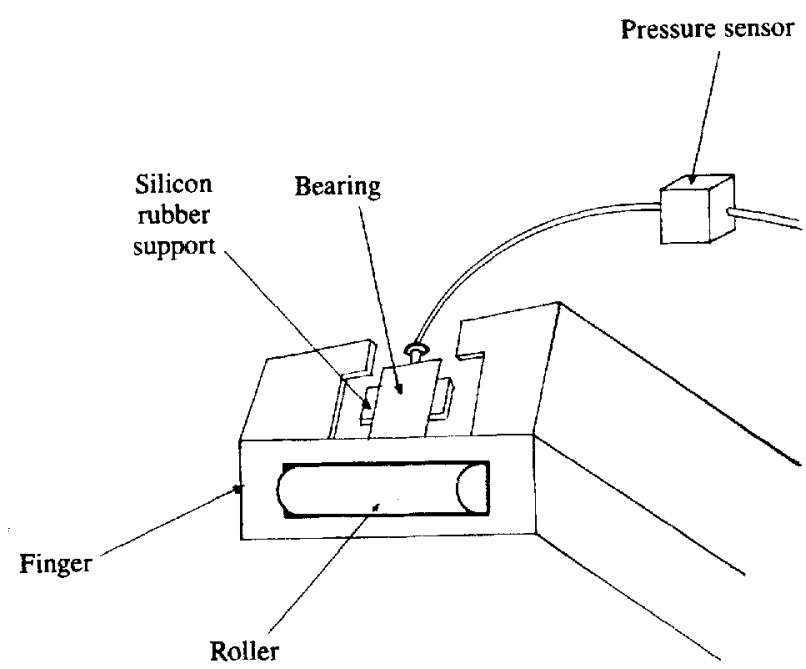

Fig. 4 A roller-type slip detecting sensor 


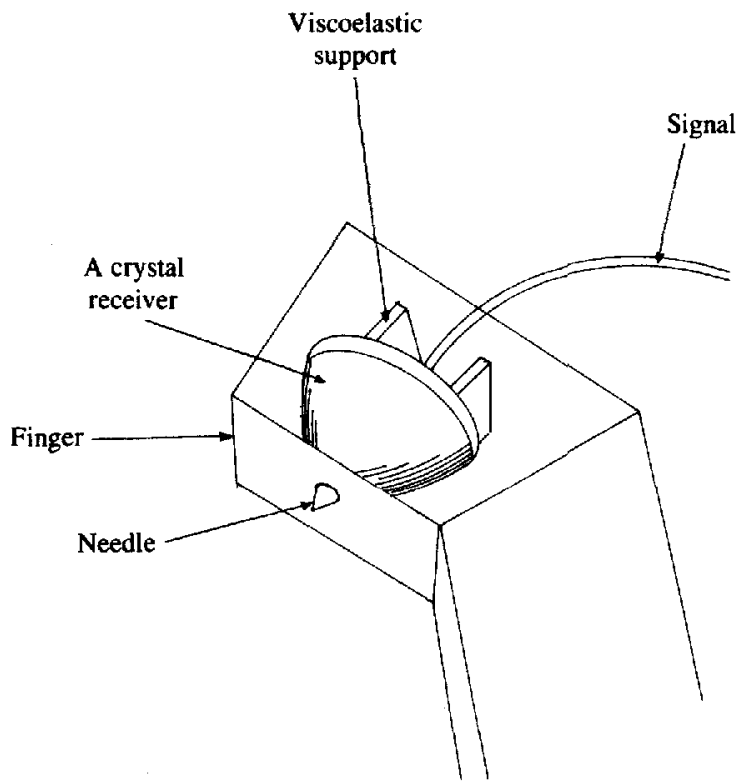

Fig. 5 A slip detecting piezoelectric contact sensor

slip monitoring in 16 directions. A 16 array of LEDs is used to indicate the direction of slip pulse; the frequency of their blinking provides a measure of slipping rate. The main limitation of roller-type slip sensors, whether with magnetic conversion or photoelectric signals is the sequential acquisition of slip directions. Another disadvantage is the relatively large size of sensing and monitoring devices.

As an alternative piezoelectric devices can be exploited to detect surface roughness of objects as an oscillation signal. Various types of crystal receivers are used which are supported by a viscoelastic membrane such as rubber to eliminate noise (Fig. 5). A rochelle salt crystal supported by a rubber damper and an attached sapphire needle to detect the surface roughness of objects is developed by Ueda et al. (29). An oscillograph is produced when a grasped object begins to slip. These types of slip sensing devices can be used to detect cracks, burrs and other manufactured surface defects.

Pressure sensors can also be employed to detect slipping of objects. One configuration described in reference (29) uses a silicon rubber pad as an elastic body incorporating a needle and backed by a layer of hard rubber. A pressure sensor semiconductor device is used to monitor the changes of pressure as the pressure exerted by the needle alters as a result of slip. A tactile pick-up to recognize the shape of an object and its surface condition, and to detect the start and the effect of sliding has been designed by Lagasse et al. (30). This device consists of a supple layer of variable electric conductivity as a function of compression. A matrix of measuring electrodes is used, each of which is connected to an output lead and a large number of conducting elements. Contact pressures are detected and their variation to sliding effect is monitored.

\section{FORCE/TORQUE SENSORS}

Most arm, wrist, hand and finger sensors used presently by industrial robots are simple position and force feedback indicators. Finger sensors in particular are almost entirely made up of primitive microswitch limit devices.

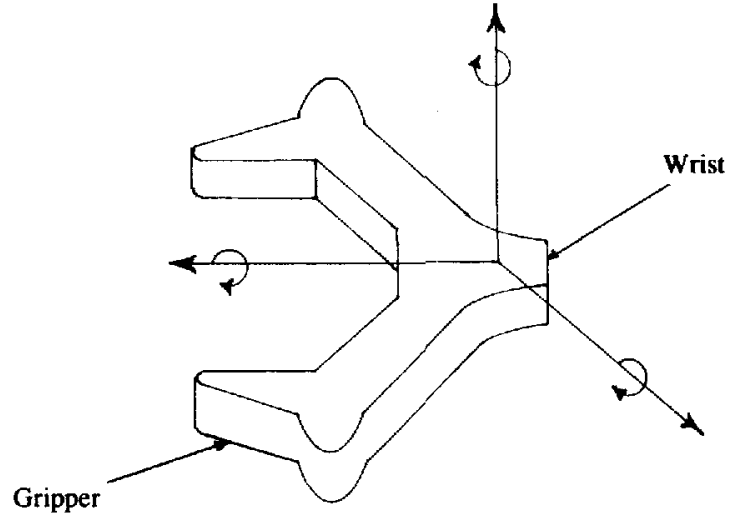

Fig. 6 Three orthogonal components of force and torque on robot wrist

However, much work has been directed to design and develop sophisticated multiple force monitoring systems to simulate the human senses of proprioception. The main desirable feature of a force/torque sensor is the measurement of three perpendicular components of force and torque on the wrist/hand of a robotic manipulator (Fig. 6).

Major contributions in development of a versatile wrist force sensor include the two rigid rings joined by three light strips each carrying a strain gauge and a strain gauge shear bridge, proposed by Nevins of Draper Laboratory (23). Another configuration uses sensitive elements consisting of semiconductor strain gauges mounted on the four deflection bars of a Maltese-shaped cross (28). This sensor resolves the gripping forces and torques along three orthogonal axes.

Electro-optical sensing elements arranged in four equidistant blocks have been used as a force/torque sensor (31). Each block contains a number of LEDs and phototransistor light paths which are interrupted by attached pins to the manipulator hand. The movements of pins alter the high-sensitive areas of phototransistors and generate corresponding electrical signals for the three components of force and torque. Two other force sensing methods have been deployed at Stanford Research Institute, one using two-member (inner and outer) mechanical supports with the end-effector fixed to the inner and the latter suspended from the outer by 16 compliant elements $(32,33)$. The relative motion between the inner and outer members is sensed by linear potentiometers and corresponds directly to the forces and torques exerted on the end-effector. The second method utilizes foil strain gauges that are cemented to several elastically flexing beams (34). Reference (34) also describes a pedestal force sensor made up of three aluminium plates, with the middle one connected to the top plate via four strain gauge force transducers which are positioned vertically. The middle plate is also connected to the bottom plate by four similar transducers that are positioned horizontally. This sensor measures the three components of force and the three components of torque that grip a part mounted on the pedestal.

Another force/torque measuring wrist sensor of lowresolution output employing ball joint mounts and strain gauges has been developed at Draper Laboratory (35). This sensor was used to steer a teleoperator system and suffered from a hysteresis effect in the ball joints. In 
general most types of force/torque sensors described so far are fairly simple arrangements of strain gauge transducers that are very reliable and inexpensive but rugged and fairly inaccurate. Furthermore, they enjoy the small compliance that is needed to effect precision assembly tasks such as fine alignment and insertion work. In addition some degree of compliance is always desirable to limit forces in non-gripping operations. Various compliant wrist sensors developed for these purposes are described in references (32) and (33). Active force sensing at a wrist and passive compliance such as remote-centre compliance somewhat pave the way in tackling close tolerance assembly operations (36). This wrist force sensory system with cross springs and strain gauges was used to effect the insertion of a 0.5 in diameter polished cylinder into a vertical hole with $7-20 \mu \mathrm{m}$ clearance in approximately three seconds. Both active and passive compliance concepts are used in this operation. The former senses forces that ensure correct manipulator position and orientation with respect to the vertical hole. The latter (passive compliance) monitors and corrects the final errors in position and orientation of the manipulator.

An adaptive active compliant force feedback system is presented by Van Brussel et al. (37) for a universal fivedegrees-of-freedom wrist. This sensory feedback system employs software-controllable active compliances that control and adjust servo gains of all axes d.c. motors with tachometer and encoder feedback. It also controls the monitored torque saturation level. This type of device overcomes the limitation of inflexible passive compliant systems such as the remote-centre compliance (RCC). However, passive devices such as RCC with predetermined compliance can deal with positional and alignment errors without the need for an on-line control system when employed for a particular application (38-40).

\section{CONDUCTIVE MATRICES AND SEMICONDUCTOR SENSORS}

Pressure and force sensing are also achieved through the use of pressure-sensitive pads, conductive materials, arrays of electrodes in elastomers and semiconductor integrated circuit (IC) transducers.

The simplest configuration of these is an array of microswitches rather like calculator buttons or a series of spring-loaded needles (41). However, the inherent threshold nature of switches constitutes loss of detail about the surface being sensed. Another sensing array of probes, each with a co-ordinating winding, monitors a series of induced e.m.f. magnitudes as the probes are displaced in accordance with the inward motion of the sensor (42).

Conductive coatings are applied to printed circuits with a series of isolated sensitive points that are uniformly distributed in a matrix (43). The imprint left by an object provides analogue information depending on the pressure exerted on every sensitive point of the coating. The choice of materials for conductive elements is rather limited. In most applications conductive rubbers, foams, some plastics and polymers are employed (44, 45) (Fig. 7). However, there are various problems with these materials, mainly hysteresis, low sensitivity, fatigue, noise and non-linearity $(46,47)$.

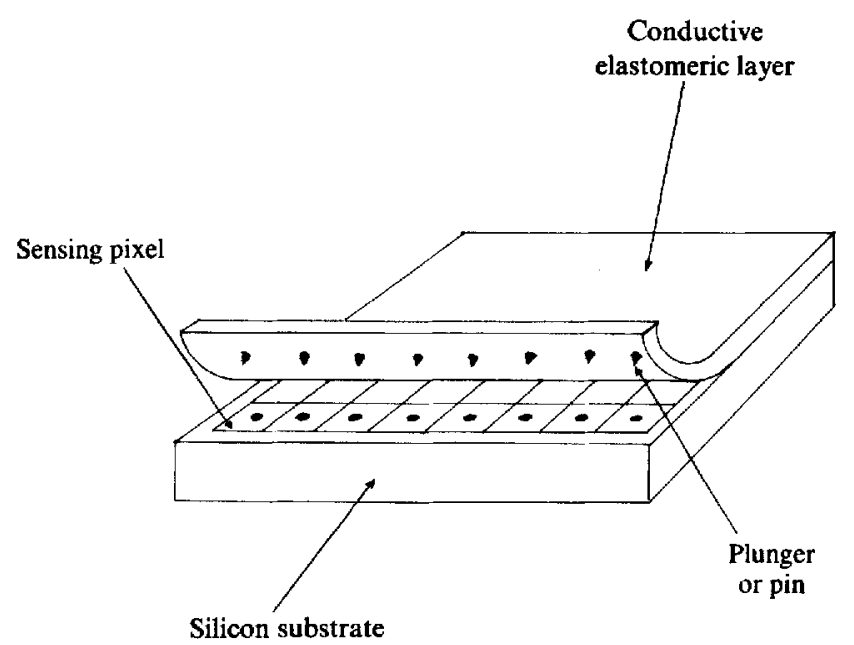

Fig. 7 A pressure-sensitive conductive-type tactile sensing array

Reference (48) describes an elastomeric pressure sensor. The elastomer is laid over a printed circuit board [similar to (43)] which is etched by 16 pairs of concentric rings. A pair of rings forms a sensing element in this configuration. Four types of elastomer are tried as sensing elements. Three types of Dynacon materials consisting of silicon rubber mixed with metallic compounds are used. All show susceptibility to high-level noise and drifting conductivity at zero pressure to varying extents.

An array of $8 \times 8$ force sensing pixels located on 0.30 in centres has been developed by Lord Corporation using an elastomeric pad surface (49). The composition of the sensor surface determines its force/deflection characteristics. Underneath each sensing pixel a plunger is located which modulates the intensity of an LED, the output of which is received by a photodiode and is proportional to the amount of plunger deflection.

A new type of tactile sensor has been designed around a VLSI (very large scale integration) device that handles tactile transduction, computing and communication. Forces are transduced by employing a standard conductive pressure-sensitive plastic. A $6 \times 3$ array of $1 \mathrm{~mm}$ square tactile cells are fabricated for such a device in the work reported by reference (50). A silicon substrate is employed and a protective glass layer is used to insulate the computing elements from the conductive plastic layer. The main advantages of this method over other sensing configurations are the elimination of parallel signal monitoring and remote data handling. In addition, larger devices incorporating a $30 \times 30$ array of electrodes can be used to provide a high-resolution tactile image of contacting objects. Comprehensive details about this type of tactile sensing architecture are available in reference $(\mathbf{5 1})$.

Another approach outlined in reference (52) utilizes a piezoelectric polymer instead of more common piezoresistive elastomers. The polymer used is $\mathrm{PVF}_{2}$ (polyvinylidene fluoride) which is light, tough and cheap. A matrix configuration of this is employed with a stiff backing and a $100 \mu \mathrm{m}$ thick $\mathrm{PVF}_{2}$ film. Contacting objects are identified by the on-off reading of the matrix. A $16 \times 16$ circular sensor, each with $6 \mathrm{~mm}$ working diameter and placed at $8 \mathrm{~mm}$ intervals, provides good spatial tactile resolution. The main advantages of this type of matrix sensor are its robustness, its 
electrically shielded structure and insensitivity to sudden changes in temperature incursions. However, to ensure for the last mentioned attribute a thick, soft rubber support sheet is required. Further reductions for temperature sensitivity and overall recognition force magnitudes are attainable by incorporating multipinned plates between the object and the $\mathrm{PVF}_{2}$ matrix. There are a number of disadvantages, one corresponding to the charge leakage from piezoelectric materials (53). Good parallelism is also required between the tactile matrix sensor and the upper pressing plate (54).

\section{THERMAL TACTILE SENSORS}

Thermally conductive materials can also be employed to distinguish between different types of material (18). For example, metallic objects have a cooler surface temperature than their counterparts made of wood or glass when kept in the same environment. A thin layer of thermally conductive material such as silicon rubber is used in conjunction with a heat source (55). A $2 \times 10$ array of miniature thermistors embedded in a silicon rubber layer forms a thermal tactile sensor (56). The thermistors measure the temperature drop caused by the flow of heat to the object through its contacting domain with the gripper jaws or fingers.

There are still a number of problems associated with this type of sensor which limit their area of application. Firstly, they seem to be most appropriate for reliable estimation of materials constitution rather than more conventional tasks of recognition of similar parts made out of similar materials. Secondly, there exists a certain degree of parasitic heat conduction and path diversions (57). Thirdly, a delay period of several seconds exists before the information from the contact area can be retrieved and processed. This delay is dependent on the dimensions of the heat detecting array. Smaller thermistors provide a faster response.

\section{TECHNICAL AND ECONOMICAL CONSIDERATIONS}

There are a number of important considerations in design and development of tactile sensors. These include technical aspects such as sensor programming within a heirarchical control structure of a flexible manufacturing environment as well as reliable signal monitoring. In addition, cost considerations are particularly important especially if the developed systems are to obtain some degree of industrial acceptance.

The former have been the subject of much research and the degree of complexity is dependent on the application and the type of tactile sensor used. Much of the research carried out so far on the software development has been in conjunction with the use of vision systems. The review of these, however, is beyond the scope of this paper and the representative literature is provided by references (58-61). Programmable sensor mediation using combined visual and tactile perception emulates human 'hand-eye' co-ordination and promises to be an important research area due to its potential applications in the stable handling of awkward objects or tools and precision assembly related tasks. For such systems an enhanced heirarchical approach is required to speed the operation cycle time of jobs $(62,63)$. The trend is to develop two-dimensional visual information related to an overhead view of an object supplemented by tactile imaging in transverse planes $(7,49)$. This eliminates the need for direct three-dimensional measurement and recognition either by visual means or a tactile device, both of which require some controlled scanning sequence of manoeuvres.

Aside from the problems of data processing and communication, cost considerations are of primary importance in the development of commercially attractive sensing devices. Most simple touch sensing configurations with low spatial resolution lend themselves to industrial applications where primitive sensory perception is deemed adequate. The typical cost of such devices is about several hundred pounds as opposed to viable alternative visual feedback systems which require an average investment of several thousand pounds. Higher-resolution tactile sensors with force, torque or pressure feedback are commercially available at slightly lower prices, but these require a greater effort in programming and data communication. Therefore, piezoresistive and piezoelectric arrays have not yet made an impact in the industrial environment, although with the development of new materials they have shown considerable potential in research.

The cost of an array of 16 miniature IC pressure sensors (employed in the design shown in Fig. 3) is merely $£ 600$ inclusive of power supply units and signal conditioning circuitry. The total cost of this design is far less than the elastomeric devices, with some added advantages. These include simple sensor programming and wear avoidance since the sensor is used as a "neartactile' device.

In general, with the decreasing cost of electronic instrumentation such as multiplexing circuits, analogue/ digital converters and continual improvement in IC techniques the tactile sensing arrays are rapidly approaching commercial viability.

\section{REFERENCES}

1 Johnson, H. A., Verderse, A. J. and Hansen, R. J. A 'smart' multimission unmanned free swimming submersible. Naval Engineer's Journal, April 1976, 88(2).

2 Engelberger, J. F. Robotics in practice, 1980 (Kogan Page, London).

3 Ernst, H. A. MH-1, a computer-operated mechanical hand. AFIPS Conference Proceedings, San Francisco, May 1962.

4 Rosen, C. A. and Nitzan, D. Use of sensors in programmabie automation. Proceedings of the Fourth International Conference on Robot Vision and Sensory Controls, October 1984.

5 Thring, M. W. Robots and telechirs, 1983 (Ellis Horwood, Chichester).

6 Soska, G. V. Third-generation robots: their definition, characteristics and application. Robotics Age, May 1985.

7 Luo, R. C., Tsai, W. H. and Lin, J. C. Object recognition with combined tactile and visual information. Proceedings of the Fourth International Conference on Robot Vision and Sensory Controls, October 1984.

8 Dorf, R. C. Robotics and Automated Manufacturing, 1983 (Reston Publishers, Peston, Virginia).

9 Heginbotham, W. B., Kichin, P. W. and Pugh, A. Visual feedback applied to programmable assembly machines. Proceedings of Second International Symposium on Industrial Robotics, Chicago, May 1972.

10 Yachida, M. and Tsuji, S. A machine vision for complex industrial parts with learning capacity. Proceedings of Fourth International Joint Conference on Artificial Intelligence, 1975.

11 Agin, G. J. and Duda, R. O. SRI vision research for advanced industrial automation. Proceedings of Second USA-Japan Computer Conference, 1975. 
12 Ishii, M., Sakane, S., Kakikura, M. and Mikami, Y. A new 3D sensor for teaching robot paths and environments. Proceedings of the Fourth International Conference on Robot Vision and Sensory Controls, October 1984.

13 Taylor, P. M., Selke, K. K. W. and Taylor, G. E. Closed loop control of an industrial robot using visual feedback from a sensory gripper. Proceedings of Twelfth International Symposium on Industrial Robots and Sixth International Conference in Industrial Robot Technology, Paris 1982.

14 Rosenfeld, A. and Pfaltz, J. L. Sequential operations in digital picture processing. J. Ass. Comput. Mach., Oct. 1966 14(4).

15 Agin, G. J. An experimental vision system for industrial application. Fifth International Symposium on Industrial Robots, Chicago, September 1975.

16 Kanade, T. and Sommer, T. M. An optical proximity sensor for measuring surface and orientation for robot manipulation. Proceedings of Third International Conference on Robot Vision and Sensory Controls, November 1983

17 Johnston, A. R. Proximity sensor technology for manipulator end effectors. Proceedings of Second Conference on Remotely Manned Systems, California, 1975.

18 Harmon, L. D. Automated tactile sensing. Int. J. Robotics Res., $1982,1(2)$.

19 Harmon, L. D. Touch-sensing technology: a review. Proceedings of Robots $V$ Conference, October 1980.

20 Goto, T. Compact packaging by robot with tactile sensors. Proceedings of Second International Symposium on Industrial Robots, Chicago, 1972.

21 Garrison, R. L. and Wang, S. S. M. Pneumatic touch sensor. IBM Technical Disclosure Bulletin, Nov. 1973, 16(6).

22 Nevins, J. L. A scientific approach to the design of computer controlled manipulators. Report R-837, Draper Laboratory, August 1974

23 Nevins, J. L. and Whitney, D. E. Robot assembly research and its future applications. Symposium held at GM Research Laboratories, Computer Vision and Sensor Based Robots, 1979 (Plenum Press, New York)

24 Bolles, R. C. and Paul, R. The use of sensory feedback in a programmable assembly system. Stanford Artificial Intelligence Project Memo 220 Stanford University, California, October 1973.

25 Mott, D. H., Lee, M. H. and Nicholls, H. R. An experimental very high resolution tactile sensor array. Proceedings of the Fourth International Conference on Robot Vision and Sensory Controls, October 1984.

26 Masuda, R., Hasegawa, K. and Osako, K. Slip sensor of industrial robot and its application. Electrical Engineering in Japan, 1976, $96(5)$.

27 Tomovich, R. and Stojiljkovich, Z. Multifunctional terminal device with adaptive grasping force. Automatica, 1975, II.

28 Bejczy, A. K. Sensor systems for automatic grasping and object handling. International Conference on Telemanipulator for Physically Handicapped, France, September 1978.

29 Ueda, M., Iwata, K., Shimizu, T. and Sakai, I. Sensors and systems of an industrial robot. Memoirs of the Faculty of Engineering, Nagoya University, 1975, 27(2).

30 Lagasse, J. C., Falipou, J., Loubieres, A., Rabis Chong, P. Richard, J. P. and Bui, A. Tactile pick-up, United States Patent 4014217, March 1977.

31 Hill, J. W. and Sword, A. J. Manipulation based on sensor directed control: an integrated end-effector and touch sensing system. Proceedings of Seventeenth Annual Human Factor Society Convention, Washington, October 1973.

32 Rosen, C. A. and Nitzan, D. Developments in programmable automation, Manufacturing Engineering, Sept. 1975.

33 A. K. Bejczy, Effect of hand-based sensors on manipulator control performance. Mechanism and Machine Theory, 1977, 12.

34 Watson, P. C. and Drake, S. H. Pedestal and wrist force sensors for automatic assembly. Proceedings of Fifth International Symposium on Industrial Robots, Chicago, September 1975.

35 Groome, R. C. Force feedback steering of a teleoperator system Draper Laboratory, Report T575, Cambridge, Massachusetts, August 1972.

36 Goto, T., Inoyama, T. and Takeyasu, K. Precise insert operation by tactile controlled robot HT-T-HAND expert-2. Proceedings of Fourth International Symposium on Industrial Robots, Japan November 1974

37 Brussel, J. Van and Simons, J. The adaptable compliance concept and its use for automatic assembly by active force feedback accommodation. Proceedings of Ninth International Symposium on
Industrial Robots, Washington DC, 1979

38 Whitney, D. E. and Nevins, J. L. What is the remote centre compliance (RCC) and what can it do? Proceedings of Ninth International Symposium on Industrial Robots, Washington DC, 1979.

39 Lane, J. D. Evaluation of a remote centre compliance device. Assembly Automation, November 1980, 1(1)

40 Watson, P. C. The remote centre compliance system and its application to high speed robot assemblies. Society of Manufacturing Engineers, paper AD77-718, 1977.

41 Will, P. M. Computer controlled mechanical assembly. Proceedings of Fifth International Symposium on Industrial Robots Chicago, 1975.

42 Page, C. J., Pugh, A. and Heginbotham, W. B. New techniques for tactile imaging. Radio and Electronic Engineer, November 1976 46(ii).

43 Briot, M. The utilisation of an artificial skin sensor for the identification of solid objects. Proceedings of the Ninth International Symposium on Industrial Robots, Washington, DC, 1979.

44 Larcombe, M. H. E. Carbon fibre tactile sensors. Proceedings of the First International Conference on Robot Vision and Sensory Controls, April 1981.

45 Purbrick, J. A. A force transducer employing conductive silicone rubber. Proceedings of the First International Conference on Robot Vision and Sensory Controls, April 1981.

46 Nitzan, D. Assessment of robotic sensors. Proceedings of the First International Conference on Robot Vision and Sensory Controls April 1981

47 Harmon, L. D. A sense of touch begins to gather momentum Sensor Review, April 1981.

48 Snyder, W. E. and St Clair, J. Conductive elastomers as sensor for industrial parts handling equipment. IEEE Trans., Instrumn Measmnt, March 1978, IM-27. (1)

49 Didocha, R. J., Lyons, D. W. and Thompson, J. C. Integration of tactile sensors and machine vision for control of robotic manipulators. Robots-9, Conference Proceedings Advancing Applications, Vol. 1, Detroit, June 1985

50 Raibert, M. H. and Tanner, J. E. A VLSI tactile array sensor Proceedings of Twelfth International Symposium on Industrial Robots and Sixth International Conference on Industrial Robot Technology, Paris, 1982.

51 Tanner, J. E., Eskenazi, R. and Raibert, M. H. A VLSI tactile sensing array computer. Caltech Conference on VLSI Systems, Pasadena, 1981.

52 Dario, P., Bardelli, R., deRossi, D., Wang, L. R. and Pinotti, P. C Touch sensitive polymer skin uses piezoelectric properties to recognise orientation of objects. Sensor Review, Oct. 1982

53 Day, G. W., Hamilton, C. A., Gruzensky, P. M. and Phelan Jr, R. J. Performance and characteristics of polyvinylidene fluoride pyroelectric detectors. Ferroelectrics, 1976, 10

54 Dario, P., Domenici, C., Bardelli, R., deRossi, D. and Pinotti, P. C. Piezoelectric polymers: new sensor materials for robotic applications. Proceedings of Thirteenth International Symposium on Industrial Robots, Chicago, 1983.

55 Russel, R. A. Thermal touch sensing. J. Elect. Electron. Engng Inst Austral., 1984, 4(1).

56 Russel, R. A. A thermal sensor array to provide tactile feedback for robots. Int. J. Robotics Res., 1985, 4(3).

57 Hillis, W. D. A high-resolution image touch sensor. Int. J. Robotics Res., 1982, 1(2).

58 Stanescu, A. M., Banu, G. V., Vlad, V. I., Borangiu, Th. and Serbanati, L. D. Architecture, control and software of a modular robot system with pneumatic stepping motors and vision. Proceedings of Twelfth International Symposium on Industrial Robots and Sixth International Conference on Industrial Robot Technology, Paris June 1982.

59 Hasegawa, T. A new approach to teaching object description for a manipulation environment. Proceedings of Twelfth International Symposium on Industrial Robots and Sixth International Conference on Industrial Robot Technology, Paris, June 1982.

60 Foulloy, L. P. and Kelley, R. B. Improving the precision of a robot. IEEE International Conference on Robotics, 1984.

61 Prajoux, R. Robotics research in France, Robotics Age, Spring 1980.

62 Oshima, M. and Shirai, Y. Object recognition using threedimensional information. IEEE Trans., Pattern Recognition Annals of Machine Intelligence, July 1983, PAMI-5(4)

$63 \mathrm{Hu}, M . K$. Visual pattern recognition by moment invariants. IRE Trans. Information Theory, Feb. 1962, IT-8. 